15

\title{
Магнитно-импульсное деформирование сплава TiNi: эксперимент и расчет
}

\author{
(C) Е.С. Остропико, С.Г. Магазинов, С.И. Кривошеев
}

Санкт-Петербургский политехнический университет Петра Великого, 195251 Санкт-Петербург, Россия

e-mail: es-ostropiko@mail.ru, magazinov_sg@mail.ru, ksi.mgd@gmail.com

Поступило в Редакцию 30 августа 2021 г.

В окончательной редакции 6 октября 2021 r.

Принято к публикации 7 октября 2021 г.

Методы магнитно-импульсного нагружения известны еще с 80-х годов XX века и, как правило, применяются для определения закономерностей разрушения материалов под действием импульсов длительностью в несколько микросекунд. В работе использована модифицированная схема магнитно-импульсной установки для высокоскоростного одноосного растяжения. Показано применение схемы на образцах из сплава TiNi c возможностью экспериментального измерения времени накопления деформации, скорости деформирования. Представлены результаты конечно-элементного моделирования и аналитического решения. Оба подхода продемонстрировали хорошее соответствие расчетной остаточной деформации экспериментальным данным, даже на образцах из сплава TiNi со специфической диаграммой деформирования. Аналитическое решение показало хорошее качественное соответствие в оценке времени накопления деформации. На основе аналитического решения проведена оценка возможностей применения магнитно-импульсного способа нагружения для одноосного высокоскоростного деформирования металлов.

Ключевые слова: магнитно-импульсное нагружение, высокоскоростное деформирование, сплав TiNi.

DOI: $10.21883 / J T F .2022 .01 .51868 .247-21$

\section{Введение}

Магнитно-импульсные методы используются для создания управляемых импульсов давления микросекундной длительности [1-3]. Этот класс методов известен еще с 80-х годов [4] и обычно используется для выявления закономерностей при высокоскоростном разрушении материалов [5,6]. В качестве особенности можно отметить формирование в материале напряженного состояния без предварительной нагрузки, что может быть важно для материалов с пространственной анизотропией их деформационных характеристик, таких как композиты, слоистые материалы и некоторые металлы [7-10]. Ранее для исследования свойств сплава с памятью формы $\mathrm{TiNi}$ после высокоскоростного нагружения для реализации магнитно-импульсного деформирования в режиме растяжения была адаптирована одна из схем [11]. Было показано, что этот подход может быть использован для высокоскоростного одноосного деформирования материалов в режиме прямого растяжения и исследования свойств, не связанных с разрушением.

Сплавы с памятью формы обладают набором уникальных свойств: высокой прочностью, коррозионной стойкостью, биосовместимостью, демпфирующей способностью и, самое главное, формовосстановлением. При нагревании они способны частично или полностью вернуться к предварительно заданной форме. Механизм этого свойства обусловлен термоупругим обратимым мартенситным фазовым превращением [12]. Известно, что функциональные свойства сплавов с памятью фор- мы зависят от формы предварительного нагружения и скорости деформации. Много исследований посвящено влиянию скорости деформирования на механические свойства и структуру сплавов [13-18], на исследование псевдоупругого поведения [19-22] и на проявление функциональных свойств [23-26]. Почти все исследования связаны с испытаниями на сжатие. Причина ясна наиболее распространенным методом высокоскоростного деформирования является метод Кольского [27]. Конечно, есть модификации этого метода для испытаний на растяжение [28], но к ним прибегают нечасто, так как они требуют специфической подготовки образцов и трудоемкой обработки результатов, допускающих неоднозначную трактовку. Даже в испытаниях на сжатие время накопления деформации составляет 100-200 $\mu \mathrm{s}$, a скорость деформирования обычно не превышает $1000-1500 \mathrm{~s}^{-1}$. Найти работы с более высокими скоростями деформирования, чтобы при этом испытуемый образец не разрушался, практически невозможно. Но однозначно можно утверждать: вопрос влияния скорости нагружения на поведение сплавов с памятью формы и металлов в целом пользуется большим интересом со стороны исследователей.

Цели настоящей работы - продемонстрировать схему магнитно-импульсного нагружения, модифицированную для одноосного растяжения с возможностью экспериментального измерения времени накопления деформации, сравнить экспериментальные данные (величину остаточной деформации, время накопления деформации, скорость деформирования) с результатами конечно- 
элементного моделирования и аналитической оценки для сплава TiNi в целях проверки их прогностической способности, провести аналитический анализ нагружающей схемы и выявить возможные закономерности.

\section{1. Экспериментальная процедура}

Схема экспериментальной установки и размеры образцов, использованных в испытаниях, представлены на рис. 1. Установка состоит из генератора импульсного тока (ГИТ) и пары магнитно-импульсных драйверов (МИД) - плоских медных шин толщиной $0.3 \mathrm{~mm}$ и шириной $4 \mathrm{~mm}$, которые располагаются в разрезах образца особой формы. $C, L, R$ - емкость, индуктивность и сопротивление генератора, а $S$ - разрядное устройство. Генератор создает ток $I$ за счет разряда конденсаторной батареи $C$. В классической схеме ток протекает через МИД, вставленный в разрез образца с макродефектом типа трещины [4]. В схеме для одноосного растяжения ток распараллеливается на два МИД, вставленных симметрично в разрезы специально подготовленного образца. Пространство между драйвером и образцом, как и пространство внутри петли драйвера, заполняется диэлектриком. Между токоведущими шинами каждого МИД генерируется магнитное поле, параметры которого (форма, амплитуда, длительность) однозначно определяются параметрами тока. На шины действует магнитное давление $P_{M}$, которое прикладывается симметрично по обе стороны от рабочей части образца к берегам разреза и приводит к одноосному растяжению рабочей части. Импульс тока $I(t)$ измеряется с помощью осциллографа поясом Роговского, параметры которого определяются в соответствии с правилами, изложенными в [29]. В работе использовали ГИТ с зарядным напряжением $U=50 \mathrm{kV}$, емкостью $C=14.8 \mu \mathrm{F}$ и индуктивностью $L=80 \mathrm{nH}$.

Измерение скорости перемещения образца производилось с использованием лазерной методики, основанной на измерении интенсивности лазерного излучения при перекрытии оптического пути торцом(краем) образца.



Рис. 1. Схема магнитно-импульсной установки на растяжение. Генератор импульсного тока с образцом из сплава TiNi (слева). Оптическая система для оценки времени накопления деформации (справа). I - лазер, II - световод. Рабочая часть образца и ее размеры отмечены красным (в онлайн версии).
Лазер, частично направленный на торец образца, за которым находится световод, через преобразователь подключен к осциллографу. В процессе деформирования торец смещается и перекрывает световод, интенсивность потока уменьшается, сигнал на осциллографе уменьшается.

Визуализация процесса разрушения с использованием высокоскоростной камеры (Photron FASTCAM SA5) подтвердила допущение о симметричном перемещении (в стороны от драйверов) возбуждаемых импульсным давлением частей образца. Этот эффект наблюдался не только при деформировании до заданной остаточной деформации, но и сохранялся до момента разрушения образца. Симметричность процесса деформирования обусловила использование лазерной методики.

Меняя параметры ГИТ, можно варьировать параметры импульса тока - его частоту и коэффициент затухания. Меняя заряд конденсатора, можно варьировать амплитуду импульса. В наших испытаниях импульс представляет затухающую синусоиду с отношением соседних амплитуд $\sim 0.6$ и периодом 6-7 $\mu$ s (рис. $2, a)$.

Магнитное давление (рис. $2, b)$ определяется по формуле [4,5]:

$$
P_{m}=k \frac{\mu}{2}\left(\frac{I}{b}\right)^{2},
$$

где $\mu=4 \pi 10^{-7} \mathrm{H} / \mathrm{m}-$ магнитная постоянная, $I-$ ток, протекающий через проводник, $b$ - ширина проводника, а $k$ - поправочный коэффициент, связанный с геометрией драйверов и тем фактом, что с увеличением частоты распределение тока становится неравномерным по ширине шин драйвера. В рассматриваемом случае $k \approx 0.42[5]$.

Образцы были изготовлены с помощью электроэрозионной резки из пластины толщиной $2 \mathrm{~mm}$. Сечение рабочей части - $1 \mathrm{~mm}^{2}$, высота - $2.5 \mathrm{~mm}$. Высота нерабочих частей - $6 \mathrm{~mm}$, длина - $30 \mathrm{~mm}$. Масса образцов $\sim 5 \mathrm{~g}$. Такие образцы сохранили симметрию и плоскопараллельность нерабочих участков во всех проведенных испытаниях, вплоть до разрыва - нерабочие участки практически не деформировались под действием магнитного давления, а вся деформация накапливалась в рабочей части.

\section{2. Аналитическая оценка и конечно-элементное моделирование}

Аналитическое описание процесса деформирования в рассматриваемой схеме нагружения было предложено ранее в [30]. В этой работе применяется тот же подход, но не для оценки максимально накопленной деформации, а для определения остаточной деформации с учетом разгрузки материала. Важно отметить, что такое решение верно только для образцов, у которых нерабочая часть не деформируется, т. е. весь импульс направлен на 

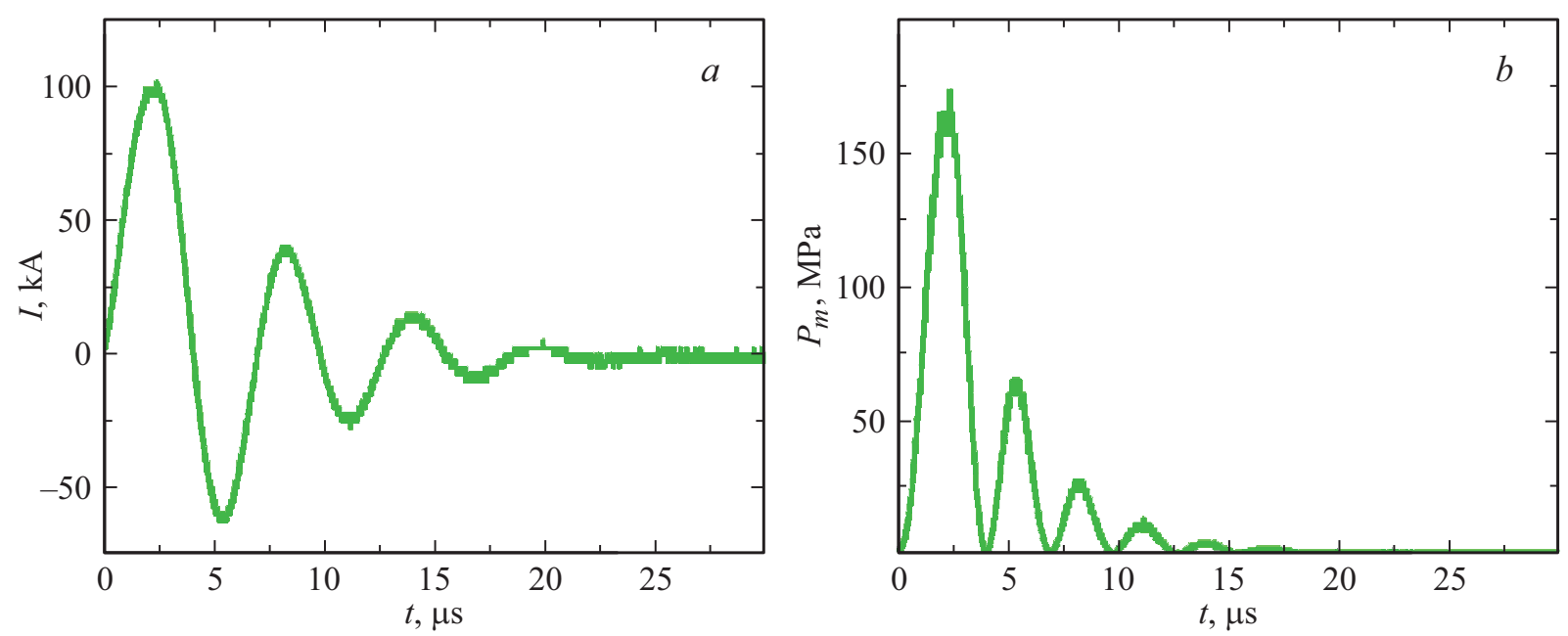

Рис. 2. Пример осциллограммы импульса тока $I(t)(a)$ и соответствующее ему магнитное давление $P_{M}(t)(b)$. Из испытания до $\sim 15 \%$ остаточной деформации.
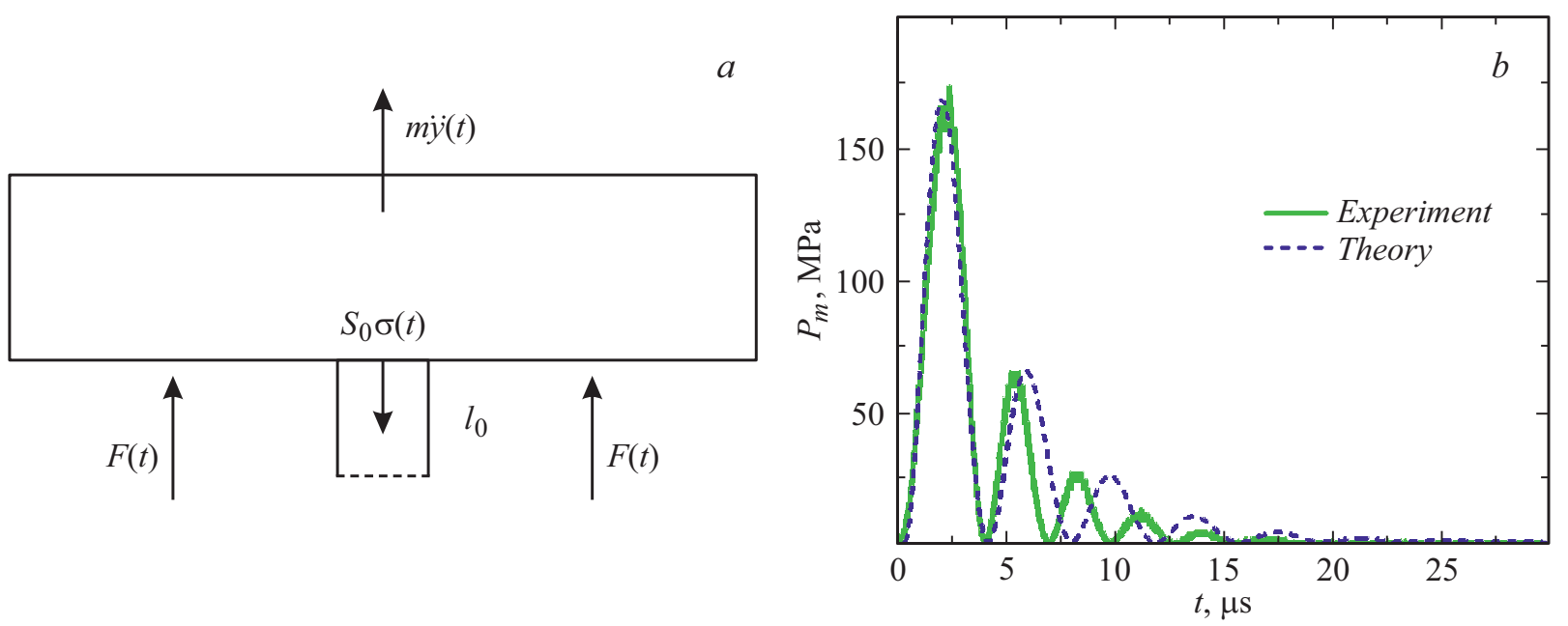

Рис. 3. Схема задачи $(a)$. Экспериментальный импульс магнитного давления и его теоретическое приближение $(b)$. Из испытания до $\sim 15 \%$ остаточной деформации.

деформирование рабочей части. С этим предположением и с учетом симметрии уравнение движения выражается следующим образом:

$$
m \ddot{y}(t)=F(t)-S_{0} \sigma(t),
$$

где $m-$ массы половины образца, $S_{0}-$ площадь сечения рабочей части, $\sigma(t)$ - напряжение в рабочей части образца при растяжении (рис. $3, a$ ).

Внешняя сила $F(t)$ определяется через магнитное давление $P_{M}(t)$, которое связано с импульсом тока

$$
F(t)=S P_{M}(t)=S P \frac{\sin ^{2}(2 \pi f t)}{e^{2 t / \tau}}
$$

где $f-$ частота, $\tau$ - постоянная времени затухания, $S$ - площадь приложения магнитного давления, $P$ параметр, который подбирается так, чтобы амплитудное значение совпадало с экспериментальным (рис. $3, b$ ).
Так как в цепи ГИТ имеются нелинейные элементы (например, искровой разрядник и нагреваемые в процессе протекания тока токоведущие элементы), то форма импульса тока (давления) несколько отличается от идеальной затухающей синусоиды. Поэтому для сопоставления экспериментальных данных с результатами расчета параметры воздействия подбирались так, чтобы максимально совпадали первые два пика импульса давления, как самые энергоемкие и эффективные.

Сопротивление материала задается в кусочнолинейном приближении набором уравнений прямых на основе диаграммы деформирования рассматриваемого материала (рис. 4):

$$
\sigma(t)=a \varepsilon(t)+b=a \frac{y(t)}{l_{0}}+b,
$$

где $l_{0}-$ исходная длина рабочей части образца; $a, b$ - параметры прямолинейных участков на кусочно- 


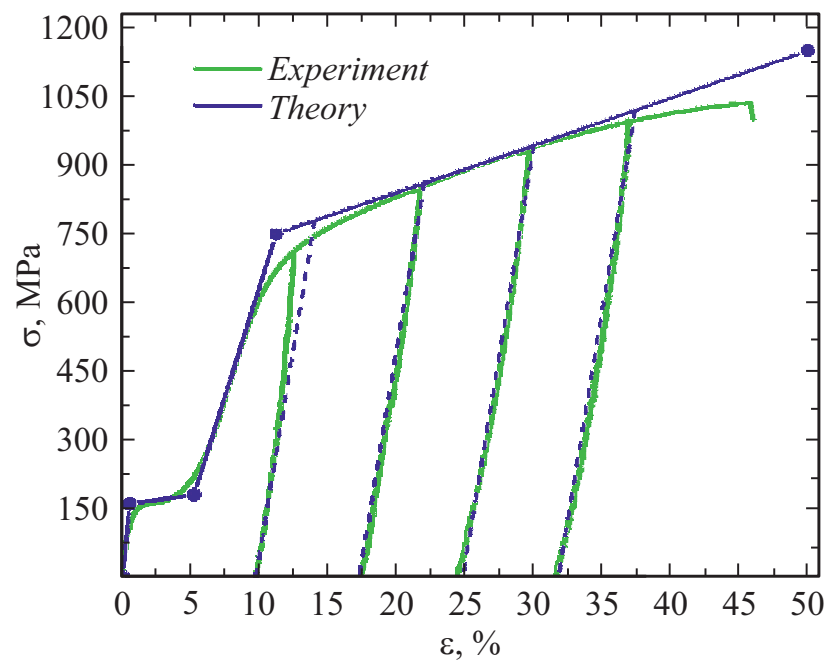

Рис. 4. Кривая деформирования сплава TiNi и ее кусочнолинейное приближение. Вершины кусочно-линейного приближения: $(0 ; 0),(0.5 ; 160),(5.2 ; 180),(11.2 ; 750),(50 ; 1150)$.

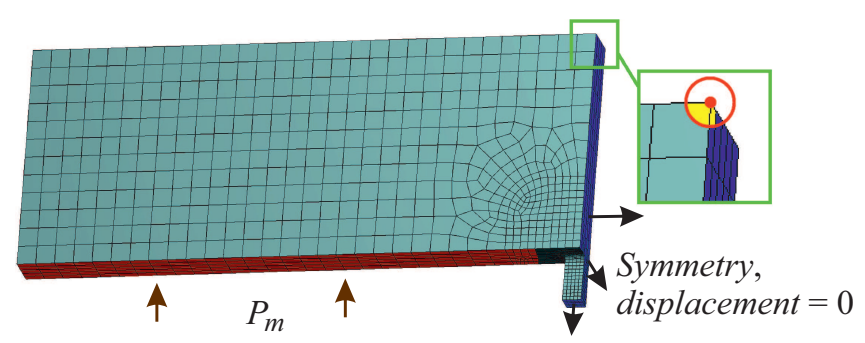

Рис. 5. Конфигурация конечно-элементной сетки, исследуемой узел.

линейном приближении, которые легко определяются по границам этих участков. Диаграмма деформирования получена с помощью универсальной испытательной машины, оснащенной видеоэкстензометром. Механическое поведение материалов с памятью формы, находящихся в низкотемпературной мартенситной фазе, отличается от привычной: после упругого участка, при достижении предела переориентации, материалы испытывают локальную пластическую деформацию, обусловленную переориентацией мартенсита. Дальнейшая форма кривой соответствует привычному поведению: следует пропорциональный участок деформирования переориентированной структуры, затем при достижении предела текучести дислокационная пластическая деформация. Безусловно, скорость нагружения влияет на величины пределов переориентации, текучести и прочности в материале: чем выше скорость, тем выше и пределы [26,31]. Однако эта разница не катастрофична, а для теоретической оценки поведения материала ее влиянием можно пренебречь.

Помимо испытания на разрыв, была проведена серия испытаний с разгрузкой. В приближении эти участки также можно представить отрезками, параллельными друг другу, что позволяет определить правило возврата деформации. Координаты вершин кусочно-линейного приближения: $(0 ; 0), \quad(0.5 ; 160), \quad(5.2 ; 180), \quad(11.2 ; 750)$, $(50 ; 1150)$.

Общее решение (2) с учетом (3) и (4) выглядит следующим образом:

$$
\begin{aligned}
& y(t)=C_{1} \sin \sqrt{A} t+C_{2} \cos \sqrt{A} t-\frac{B}{A}+\frac{F \tau^{2}}{2 A \tau^{2}+8} e^{-\frac{2 t}{\tau}}+ \\
& \frac{8 F \pi f \tau^{3}}{\left(4+A \tau^{2}-16 \pi^{2} f^{2} \tau^{2}\right)^{2}+256 \pi^{2} f^{2} \tau^{2}} e^{-\frac{2 t}{\tau}} \sin 4 \pi f t \\
& +\frac{8 F \pi^{2} f^{2} \tau^{4}-2 F \tau^{2}-\frac{F A \tau^{4}}{2}}{\left(4+A \tau^{2}-16 \pi^{2} f^{2} \tau^{2}\right)^{2}+256 \pi^{2} f^{2} \tau^{2}} e^{-\frac{2 t}{\tau}} \cos 4 \pi f t,
\end{aligned}
$$

где $A=S_{0} a / m l_{0}, B=S_{0} b / m, F=S P / m$.

Для определения $C_{1}$ и $C_{2}$ исходим из того, что в начальный момент времени $y(0)=0, \dot{y}(0)=0$, а начальные условия на последующем участке должны соответствовать значениям, достигнутым в конце предыдущего участка. После решения задач Коши на участках кусочно-линейного приближения получаем зависимости перемещения от времени. Для получения полного перемещения остается сложить приращения $y(t)$ на каждом участке в общую последовательность. Отношение $\varepsilon(t)=y(t) / l_{0}$ выражает изменение деформации во времени. Максимум этого отношения, во-первых, определяет момент накопления максимальной деформации, а вовторых момент, в который наступает линейный возврат по правилу разгрузки.

Конечно-элементное моделирование поведения материала под действием магнитно-импульсного нагружения проводили в среде ANSYS Workbench. Механические свойства материала, использованные при моделировании: плотность TiNi - $6500 \mathrm{~kg} / \mathrm{m}^{3}$, модуль Юнга $32 \mathrm{GPa}$, коэффициент Пуассона - 0.33. Правило нелинейного деформирования материала (nonlinear plasticity) было задано аналогично - как кусочно-линейное приближение диаграммы деформирования в соответствии с рис. 4.

Поскольку задача симметрична, для моделирования использовали часть образца, добавив условия симметрии и перемещения на соответствующие поверхности (рис. 5).

В качестве нагружающего импульса $P_{m}$ использовали импульс магнитного давления, полученный экспериментально. Деформацию и время накопления деформации оценивали по перемещению узла, отмеченного на рис. 5.

\section{3. Результаты расчетов и сравнение с экспериментом}

Проведя серию испытаний, мы получили набор экспериментальных величин и зависимостей: масса образца, его геометрические параметры, импульс тока $I(t)$, и зависимость, отражающую изменение интенсивности 


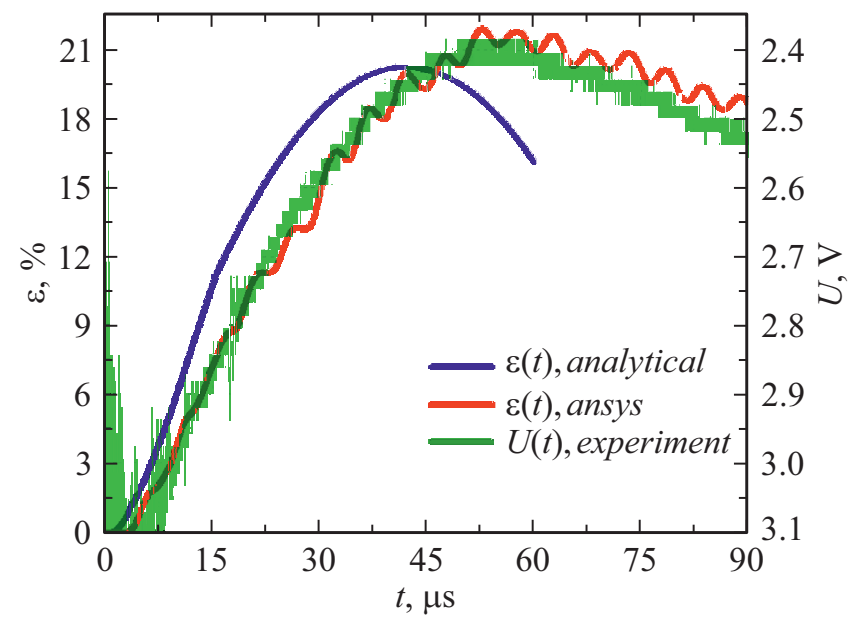

Рис. 6. Зависимости $\varepsilon(t)$, полученные при аналитическом и конечно-элементном расчете. Зависимость $U(t)$ отражает изменение интенсивность пучка света с течением времени. Испытания образца до $\sim 16 \%$ остаточной деформации.

света с течением времени $U(t)$, которая связана с перемещением торца образца под нагрузкой. По значению амплитуды $I(t)$ по (1) получили амплитуды магнитного давления: 160, 173, 200, $260 \mathrm{MPa}$. Подобрав параметры $P, f, \tau$ для (3) в каждом отдельном испытании получили аналитическое приближение нагружающих импульсов подобно рис. 3, $b$. Подставив все параметры: масса $m$, сечение рабочей части $S_{0}$, площадь поверхности нагружения $S$, начальная длина рабочей части $l_{0}$, параметры кусочно-линейных участков $a$ и $b$, параметры импульса $P, f, \tau-$ в (5), собрав приращения $y(t)$ в единую последовательность, получили кривые зависимости деформации от времени $\varepsilon(t)=y(t) / l_{0}$. Определив амплитудное значение $\varepsilon(t)$, мы смогли в линейном приближении (4), по правилу возврата деформации в соответствии с рис. 4 получить остаточную деформацию.

Результаты конечно-элементного моделирования в программном пакете ANSYS представляются в виде зависимости $y(t)$ для узла, отмеченного на рис. 5. Аналогично построена конечно-элементная зависимость деформации от времени $\varepsilon(t)=y(t) / l_{0}$.

На рис. 6 на примере образца, деформированного до $\sim 16 \%$ остаточной деформации, показаны результирующие зависимости $\varepsilon(t)$, полученные при аналитическом расчете и конечно-элементном моделировании, а также зависимость $U(t)$, отражающая изменение интенсивность света при перемещении торца образца с течением времени.

У экспериментальной зависимости $U(t)$ в момент начала испытания наблюдается шум, связанный со срабатыванием разрядника. Когда образец начинает деформироваться, он перекрывает световод, интенсивность уменьшается, а напряжение на осциллографе падает с $\sim 3.1$ до $\sim 2.4 \mathrm{~V}$, поэтому шкала перевернута. Видно, что на этапе роста деформации наблюдается качествен- ное соответствие всех трех кривых. Максимальные деформации в расчетных зависимостях хотя и близки, но конечно-элементный расчет дает немного большие значения, чем аналитический. Расчет в ANSYS учитывает волновую природу деформирования, что отражается в характере кривой $\varepsilon(t)$ - ее волновой вид с периодическими „падениями“ деформации приводит к тому, что времени накопления деформации $t_{m}$ требуется больше, по сравнению с аналитическим расчетом, это делает ее качественно ближе к экспериментальной кривой. Аналитический расчет волновую природу не учитывает, и деформация накапливается быстрее.

На рис. 7 приведены экспериментальные и расчетные зависимости остаточной деформации $\varepsilon_{\text {res }}$, времени накопления деформации $t_{m}$ и скорости пластической деформации $\dot{\varepsilon}=\varepsilon_{r e s} / t_{m}$ от амплитуды магнитного давления $P_{m}$.

Расчетные величины остаточной деформация согласуются с экспериментальными (рис. 7,a). Однако полученные в ANSYS значения остаточной деформации несколько завышены, особенно в испытаниях с малыми деформациями. По всей видимости, это связано с особенностями расчета в зоне переориентации мартенсита. С ростом деформации наблюдаемая особенность нивелируется: чем больше деформация, тем меньше доля этого участка в общей расчетной деформации, тем ближе расчетные значения к экспериментальным. Так что на больших деформациях ANSYS дает более точное соответствие эксперименту по всем измеренным параметрам.

При оценке времени накопления деформации $t_{m}$ (рис. $7, b)$, значения, определенные аналитически, меньше экспериментальных на 20-30\%. При этом наблюдается качественное соответствие. ANSYS же показывает более точные значения с разницей в 5-10\%. Как следствие, совокупность аналогичных различий присуща зависимости $\dot{\varepsilon}$, поскольку $\dot{\varepsilon}=\varepsilon_{r e s} / t_{m}$. На рис. 7, $c$ видно, что аналитический расчет завышает скорость пластической деформации в среднем на 35-40\%, но дает качественное соответствие. ANSYS в рассмотренном диапазоне испытаний дает более близкие значения $\dot{\varepsilon}$ к экспериментальным, но в качественном смысле полученные зависимости заметно отличаются. При малых значениях амплитуд импульса давления средние значения скорости пластической деформации, полученные в ANSYS, ближе к аналитическим, а при больших амплитудах импульса давления приближаются к экспериментальным.

Можно утверждать, что для первичных оценок оба подхода дают неплохое соответствие расчетных остаточных деформаций эксперименту. Время накопления деформации ANSYS показывает точнее, но, если говорить о качественном поведении материала, аналитический метод справляется не хуже и при этом проще и быстрее в исполнении.

Опираясь на качественное соответствие и прогностические способности аналитического решения, был проведен ряд расчетов по деформированию подобных об- 

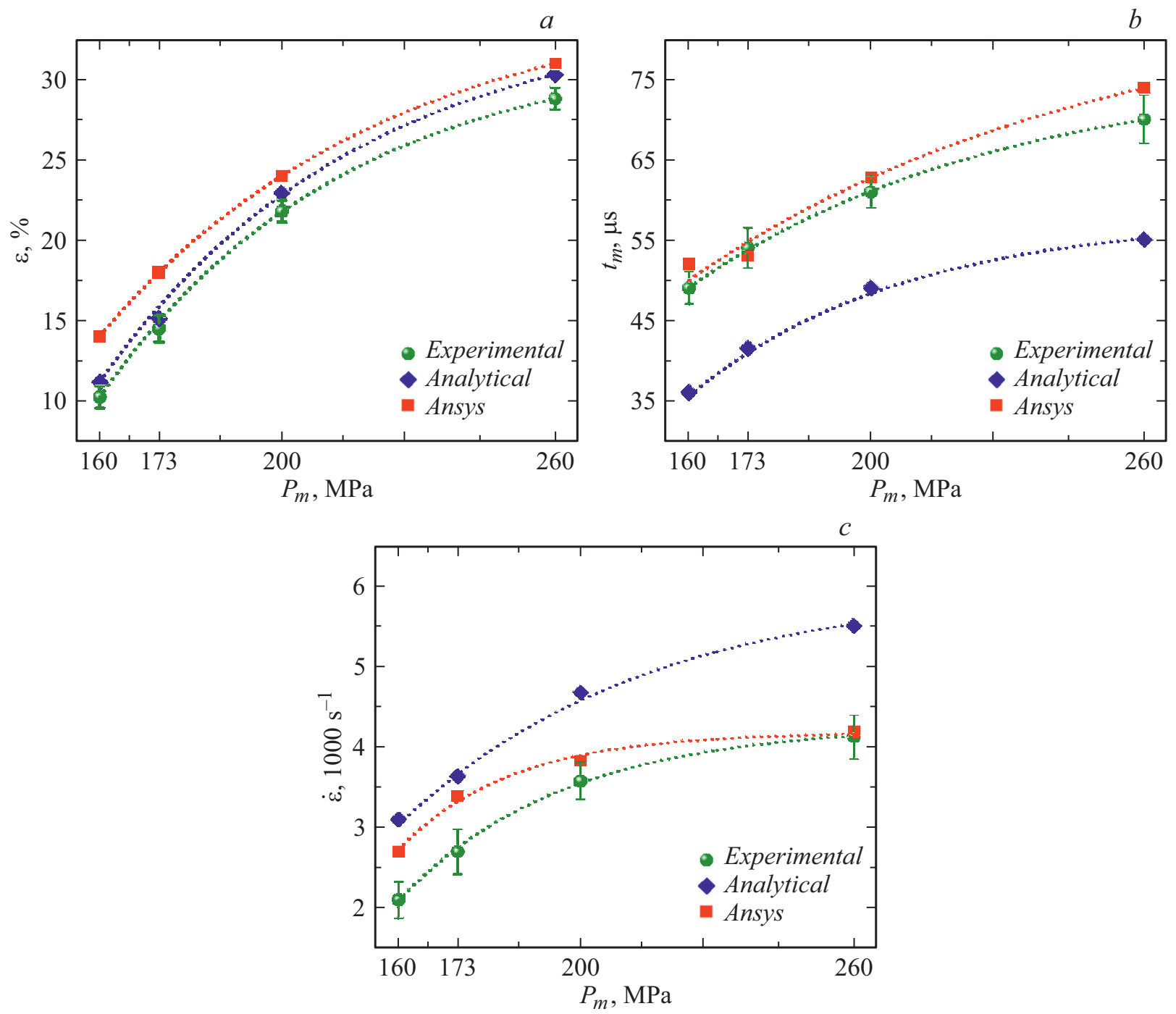

Рис. 7. Экспериментальные и расчетные зависимости: остаточной деформации $\varepsilon_{r e s}$ от амплитуды магнитного давления $P_{m}(a)$ времени накопления деформации $t_{m}$ от амплитуды магнитного давления $P_{m}(b)$, скорости пластической деформации $\varepsilon$ от амплитуды магнитного давления $P_{m}(c)$.

разцов (рис. 1) импульсами аналогичной формы (рис. 2). Для удобства положим $\varepsilon=50 \%$ как предельную деформацию материала и проанализируем, как в зависимости от частоты нагружающего импульса и массы образца меняется время накопления этой деформации $t_{m}$ (рис. $8, a$ ). Macca $m=5 \mathrm{~g}$ - масса экспериментального образца. Кривая $T / 2$ на рисунке показывает полпериода импульса соответствующей частоты.

Ожидаемо, чем меньше масса образца, тем ниже располагается кривая $t_{m}(f)$, поскольку образец менее инертен. На малых частотах нагружающего импульса время tm становится соизмеримо с $T / 2$, т. е. процесс деформирования переходит к квазистатическому режиму. С ростом частоты импульса время $t_{m}$ асимптотически стремится к некоторой величине. Следовательно, для заданного уровня деформации существует некоторое минимально возможное время накопления деформации, которое характеризуется периодом собственных колебаний рассматриваемого образца.

На частотах выше $f=80 \mathrm{kHz}$ существенного уменьшения $t_{m}$ не наблюдается. Однако на высоких частотах, чтобы накопить соответствующую деформацию, требуется существенно увеличить амплитуду $P_{m}$ (рис. $8, b$ ). Теоретически можно сказать, что для исследуемого материала диапазон частот $80-150 \mathrm{kHz}$ - оптимальный диапазон, который позволяет получить практически минимальные значения $t_{m}$, не требуя экстремальных значений магнитного давления.

Конечно, если стоит задача провести испытание на разрушение, то увеличение амплитуды $P_{m}$ приведет к более быстрому накоплению тех же $\varepsilon=50 \%$ и разрушению. На рис. $8, c$ показаны зависимости $\varepsilon(t)$ для импульсов различной амплитуды, частотой $80 \mathrm{kHz}$ $(T=12.5 \mu \mathrm{s})$. Видно, что с ростом $P_{m}$ время накопле- 

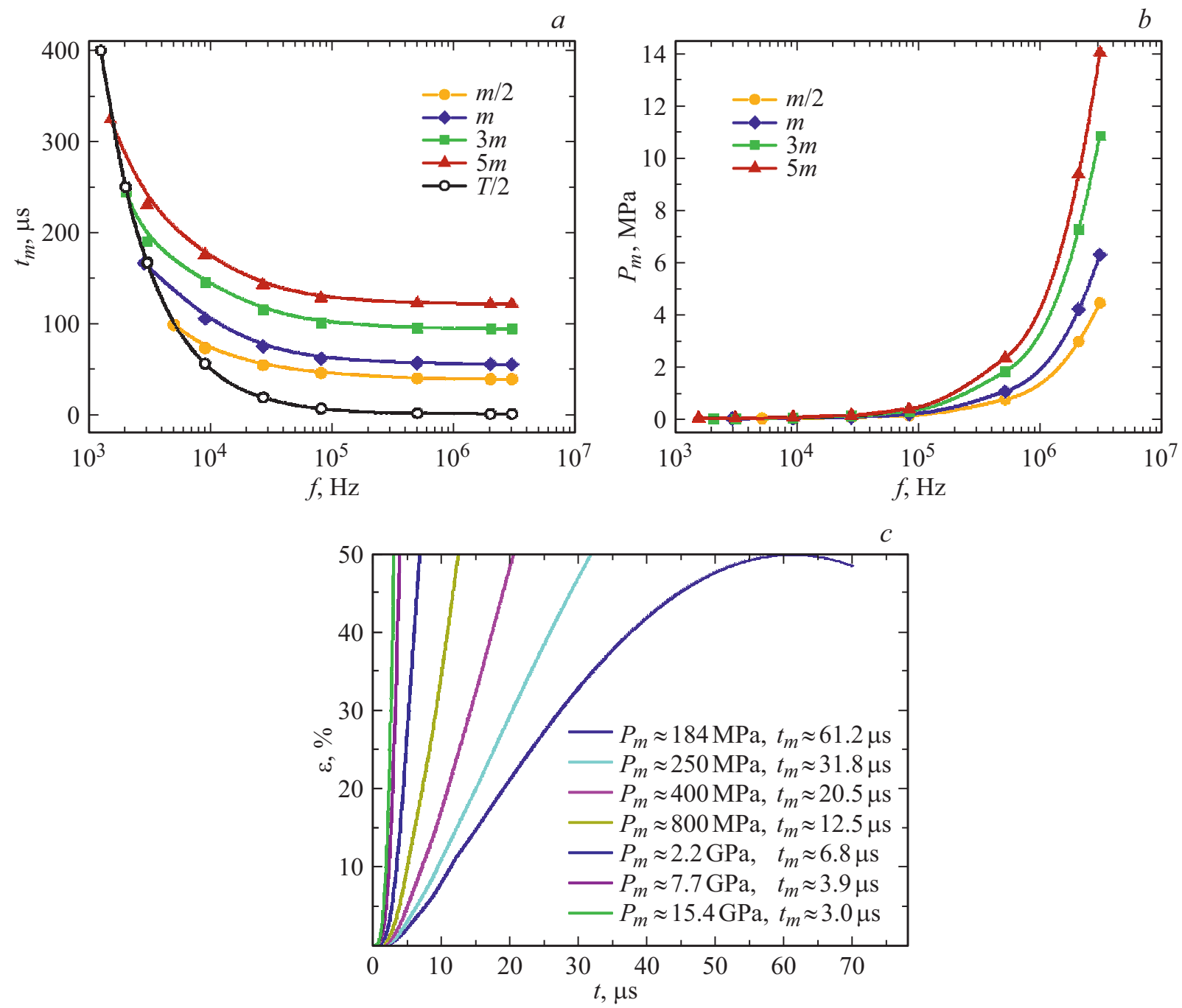

Рис. 8. Результаты аналитических расчетов. Масса $m=5 \mathrm{~g}$. Время накопления $50 \%$ деформации в зависимости от массы образца и частоты нагружающего импульса $(a)$ и соответствующая амплитуда магнитного давления $P_{m}(b)$. Зависимости $\varepsilon(t)$ для импульсов различной амплитуды частотой $80 \mathrm{kHz}(c)$.

ния $t_{m}$ становится сопоставимо с периодом нагружающего импульса. Для рассматриваемого метода нагружения ограничения в достижимых амплитудах давления связаны с индукцией магнитного поля, достижимая амплитуда которой связана с энергией сублимации материала, из которого изготовлена магнитная система, и параметрами генератора импульсного тока. В [32] было показано, что предельное значение индукции магнитного поля в магнитной системе из меди составляет 340-360 Т, при этом магнитное давление может доходить до $50 \mathrm{GPa}$.

В проведенных экспериментах частота импульса составляла $\approx 140 \mathrm{kHz}$. Максимальная достигнутая остаточная деформация составила $\approx 29 \%$, а время ее накопления $\approx 68 \mu \mathrm{s}$, т.е. скорость пластической деформации составила $\approx 4300 \mathrm{~s}^{-1}$. Судя по изложенному выше, можно предположить, что в рассмотренной схеме нагружения в испытаниях, не предполагающих разрушения исследованных образцов, это и есть почти предельные значения времени накопления деформации и скорости деформирования, а увеличить скорость деформирования можно путем уменьшения массы образца.

Безусловно, подобная аналитическая оценка не ограничена сплавом TiNi, а может быть применена к любому металлу с известной диаграммой деформирования.

\section{Заключение}

Продемонстрировано использование метода магнитно-импульсного нагружения для одноосного растяжения с возможностью экспериментального измерения времени накопления деформации на примере образцов из сплава TiNi.

Проведены аналитический и конечно-элементный расчеты остаточной деформации, времени накопления деформации и скорости деформирования. Оба подхода дают количественное соответствие расчетных остаточных деформаций эксперименту, даже на образцах из сплава 
TiNi c нестандартной диаграммой деформирования, достаточное для планирования экспериментов.

Конечно-элементный программный пакет ANSYS дает более точные значения времени накопления деформации в рассмотренном диапазоне испытаний (особенно, на больших деформациях), но, если говорить о качественном поведении материала, аналитическое решение лучше описывает зависимость средней скорости пластической деформации от амплитуды магнитного давления.

Теоретически показано, что нагружение импульсом частотой выше $80-150 \mathrm{kHz}$ нерационально, поскольку для достижения соответствующих деформаций требуется значительное увеличение амплитуды магнитного давления, но выигрыша во времени накопления деформации практически нет. Эффективнее уменьшать массу образца, либо менять конфигурацию нагружающей системы.

Расчеты показывают, что в рассмотренной схеме нагружения (конкретный образец-схема нагружения-ГИТ), для заданного уровня деформации существует некоторое минимально возможное время накопления деформации, которое характеризуется периодом собственных колебаний рассматриваемого образца. Увеличение скорости достижения заданных деформаций требует уменьшение массы рассматриваемого образца. Если же стоит задача провести испытание на разрушение, то увеличение амплитуды приведет к более быстрому накоплению предельной деформации и разрушению. Ограничения на амплитуду магнитного давления связаны только с величиной магнитной индукции (eе максимум зависит от энергии сублимации материала, из которого изготовлена система нагружения) и параметрами генератора импульсного тока.

\section{Благодарности}

Конечно-элементное моделирование в пакете ANSYS проводилось с использованием вычислительных ресурсов суперкомпьютерного центра Санкт-Петербургского политехнического университета Петра Великого.

\section{Финансирование работы}

Исследование выполнено при финансовой поддержке РФФИ в рамках научного проекта № 19-32-60035

\section{Конфликт интересов}

Авторы заявляют, что у них нет конфликта интересов.

\section{Список литературы}

[1] D.I. Alekseev, S. I. Krivosheev, S.G. Magazinov. MATEC Web Conf., 145, 05006, (2018). DOI: $10.1051 /$ matecconf $/ 201814505006$

[2] H. Ma, W. Mao, H.1. Su, H. Zhu, X. Cui, L. Huang, J. Li, M. Wu. Int. J. Mech. Sci., 209, 106712 (2021). DOI: $10.1016 /$ j.jjmecsci.2021.106712
[3] A. Gruzdkov, S. Krivosheev, Yu. Petrov, A. Razov, A. Utkin. Mater. Sci. Eng. A, 481-482, 105 (2008).

DOI: $10.1016 /$ j.msea.2007.03.113

[4] K.R. Chandar, W.G. Knauss. Int. J. Fract., 20, 209 (1982). DOI: doi.org/10.1007/BF01140336

[5] S.G. Magazinov, S.I. Krivosheev, Yu.E. Adamyan, D.I. Alekseev, V.V. Titkov, L.V. Chernenkaya. Mater. Phys. Mech., 40, 117 (2018). DOI: 10.18720/MPM.4012018_14

[6] S. Atroshenko, V. Morozov, D. Gribanov, A. Lukin, Y. Petrov. EPJ Web Conf., 94, 02014 (2015). DOI: $10.1051 /$ epjconf/20159402014

[7] G.I. Kanel, S.V. Razorenov, V.E. Fortov. Joint 20th AIRAPT 43th EHPRG (Karlsruhe, Germany, 2005), 119921.

[8] G.I. Kanel, S.V. Razorenov, G.V. Garkushin, A.S. Savinykh. J. Phys. Conf. Ser., 946, 012039 (2018). DOI: $10.1088 / 1742-6596 / 946 / 1 / 012039$

[9] Y. Meshcheryakov, A. Divakov, N. Zhigacheva, G. Konovalov. Proc. Struct. Int., 2, 477 (2016). DOI: $10.1016 /$ j.prostr.2016.06.062

[10] G.G. Savenkov, Yu.I. Meshcheryakov, B.K. Barakhtin, N.V. Lebedeva. J. Appl. Mech. Tech. Phys., 55, 896 (2014). DOI: $10.1134 / \mathrm{S} 0021894414050198$

[11] E.S. Ostropiko, S.I. Krivosheev, S.G. Magazinov. Appl. Phys. A, 127, 27 (2021). DOI: 10.1007/s00339-020-04160-7

[12] K. Otsuka, X. Ren. Progr. Mater. Sci., 50, 511 (2005). DOI: $10.1016 /$ j.pmatsci.2004.10.001

[13] V. Grigorieva, A. Danilov, A. Razov. Acta Phys. Pol., 128, 592 (2015). DOI: 10.12693/APhysPolA.128.592

[14] S.-Y. Jiang, Y.-Q. Zhang. Trans. Nonferrous Met. Soc. China., 22 (1), 90 (2012). DOI: 10.1016/S1003-6326(11)61145-X

[15] S.-Y. Jiang, Y.-Q. Zhang, Y.-N. Zhao, M. Tang, W.-L. Yi. J. Cent. South Univ., 20, 24 (2013). DOI: 10.1007/s11771-013-1454-6

[16] A.M. Bragov, L.A. Igumnov, A.Yu. Konstantinov, A.K. Lomunov, A.I. Razov. Adv. Struct. Mater., 103, 133 (2019). DOI: 10.1007/978-3-030-11665-1

[17] Y. Qiu, M.L. Young, X. Nie. Metall. Mater. Trans. A, 46, 4661 (2015). DOI: $10.1007 / \mathrm{s} 11661-015-3063-5$

[18] Y. Qiu, M.L. Young, X. Nie. Metall. Mater. Trans. A, 48, 601 (2017). DOI: $10.1007 / \mathrm{s} 11661-016-3857-0$

[19] W.W. Chen, Q. Wu, J.H. Kang, N.A. Winfree. Int. J. Solids Struct., 38 (50-51), 8989 (2001). DOI: $10.1016 / \mathrm{S} 0020-7683(01) 00165-2$

[20] S. Nemat-Nasser, W.-G. Guo. Mech. Mater., 38, 463 (2006). DOI: 10.1016/j.mechmat.2005.07.004

[21] H. Tobushi, Y. Shimeno, T. Hachisuka, K. Tanaka. Mech. Mater., 30 (2), 141 (1998) DOI: $10.1016 / \mathrm{S} 0167-6636(98) 00041-6$

[22] J. Zurbitu, R. Santamarta, C. Picornell, W.M. Gan, H.G. Brokmeier, J. Aurrekoetxea. Mat. Sc. Eng. A., 528 (2), 764 (2010). DOI: 10.1016/j.msea.2010.09.094

[23] В.А Лихачев, Ю.И. Патрикеев. Тез. докл. XXIV всесоюзного семинара „Актуальные проблемы прочности“, посвященного механике прочности материалов с новыми бункциональными свойствами (Рубежное, СССР, 1990), c. 128.

[24] S.P. Belyaev, N.F. Morozov, A.I. Razov, A.E. Volkov, L.L. Wang, S.Q. Shi, S. Gan, J. Chen, X.L. Dong. Mater Sci Forum., 394-395, 337 (2002). DOI: $10.4028 / w w w . s c i e n t i f i c . n e t / M S F .394-395.337$

[25] E.S. Ostropiko, A.Y. Konstantinov. Lett. Mater., 11 (2), 223 (2021). DOI: 10.22226/2410-3535-2021-2-223-228 
[26] E.S. Ostropiko, A.Y. Konstantinov. Mater. Sci. Technol., 37 (4), 1 (2021). DOI: 10.1080/02670836.2021.1958466

[27] H. Kolsky. Proc. Phys. Soc. London Sect. B., 62 (11), 676 (2002). DOI: 10.1088/0370-1301/62/11/302

[28] T. Nicholas. Exp. Mech., 21 (5), 177 (1981). DOI: $10.1007 / \mathrm{BF} 02326644$

[29] Г. Кнопфель. Сверхсильные импульсные магнитные поля: методы генерации и физические эфбекты, связанные с созданием импульсных полей мегаэрстедного диапазона (Мир, М., 1972)

[30] E.S. Ostropiko, S.I. Krivosheev, S.G. Magazinov. Lett. Mater., 11 (1), 55 (2021). DOI: 10.22226/2410-3535-2021-1-55-60

[31] A.M. Bragov, A.N. Danilov, A.Yu. Konstantinov, A.K. Lomunov, A.S. Motorin, A.I. Razov. Phys. Met. Metallogr., 116 (4), 385 (2015).

DOI: $10.1134 / \mathrm{S} 0031918 X 15040031$

[32] S.I. Krivosheev. Digest Technical Papers - IEEE International Pulsed Power Conf., 2, 750 (1999).

DOI: 10.1109/PPC.1999.823622 